\title{
An Efficient Algorithm for Obtaining the Volume of a Special Kind of Pyramid and Application to Convex Polyhedra
}

\author{
By Ted Speevak
}

\begin{abstract}
An efficient method is given for obtaining the volume of a pyramid of species $n-2$ whose base is a convex polygon. The pyramid is "transformed" into a simplex whose volume is computed directly. A refinement is provided to the Cohen-Hickey method for determining volumes of convex polyhedra.
\end{abstract}

1. Introduction. Recently, some papers have appeared which give analytically exact algorithms for obtaining the volume of convex polyhedra in $\mathbb{R}^{n}$ [1], [3], [4]. These papers were motivated by practical applications: [1] arose from work on mechanizing programme analysis and the desire to determine the probability of taking a given path through a sequence of conditionals (where the Boolean expression in a conditional consists of a linear inequality); [3], [4] were in conjunction with the building of chemical stability models. The underlying methodology is similar for these algorithms: partition the polyhedron into simplices, then sum the volumes of the constituent simplices. While the approaches are direct, the work (i.e., number of calculations) increases exponentially with $n$ [1].

This paper gives an efficient method for calculating the volume of a pyramid of species $n-2$ (see p. 123 of [2]), whose base is a 2-dimensional bounded convex polygon. Hereafter, this pyramid, which is explicitly defined at the beginning of Section 2, will be referred to simply as a pyramid of species $n-2$. Efficiency decision rules are given. Then it is shown how this method can be used to improve the efficiency of the algorithms in [1], [3], [4].

Wherever it is convenient, matrix notation will be used. Boldface capital letters (e.g., A, B, C) will denote matrices, while boldface lower case letters (e.g., a, b, c) will denote vectors. Prime will indicate transposition, with row vectors always primed, while column vectors are without a prime. $\operatorname{Det}(\cdot)$ will represent the determinant of a matrix. ABS( $(\cdot)$ will denote the absolute value of a scalar. SGN $[\cdot]$ will represent the sign of a scalar. By $\mathbb{R}^{n}$, we will denote the $n$-dimensional Euclidean space. $f$ linearly independent linear equations in $\left(x_{1}, \ldots, x_{n}\right)$ define an $(n-f)$-flat, where an $r$-flat is a flat (Euclidean) space of $r$ dimensions in the sense that $r$ coordinates are needed to fix a point of the flat.

Received July 16, 1984; revised July 15, 1985.

1980 Mathematics Subject Classification. Primary 52-04, 52A25.

Key words and phrases. Pyramid of species $n-2$, simplex, convex polyhedra, volume.

(C1986 American Mathematical Society $0025-5718 / 86 \$ 1.00+\$ .25$ per page 
2. Algorithm. Let $P_{2}^{n}$ represent a 2 -dimensional bounded convex polygon in $\mathbb{R}^{n}$, whose successive adjacent distinct vertices are the vectors $\mathbf{d}_{1}, \mathbf{d}_{2}, \ldots, \mathbf{d}_{p}$ where $p \geqslant 3$. Now consider a pyramid whose base is $P_{2}^{n}$ and with vertices represented by the vectors $\mathbf{w}_{0}, \ldots, \mathbf{w}_{n-3}$ where $n \geqslant 3$, and where $\mathbf{w}_{n-3}$ does not lie in the 2-flat defined by $P_{2}^{n}, \mathbf{w}_{n-4}$ does not lie in the 3-flat defined by $P_{2}^{n} \mathbf{w}_{n-3}$, and so on. The pyramid $P_{2}^{n} \mathbf{w}_{n-3} \cdots \mathbf{w}_{0}$ is a pyramid of species $n-2$ (see p. 123 of [2]) and the constituent simplices $\left(\mathbf{w}_{0} \cdots \mathbf{w}_{n-3} \mathbf{d}_{1} \mathbf{d}_{i} \mathbf{d}_{i+1}, i=2, \ldots, p-1\right)$ are nonempty and it follows that

$$
\operatorname{Det}\left(\begin{array}{cccc}
\mathbf{w}_{0}, \ldots, \mathbf{w}_{n-3}, & \mathbf{d}_{1}, & \mathbf{d}_{i}, & \mathbf{d}_{i+1} \\
1, \ldots, 1, & 1, & 1, & 1
\end{array}\right) \neq 0, \quad \text { where } 2 \leqslant i \leqslant p-1 .
$$

Direct evaluation of the pyramid $P_{2}^{n} \mathbf{w}_{n-3} \cdots \mathbf{w}_{0}$ entails the evaluation of the $p-2$ determinants on the LHS of (1). The volume of the pyramid $P_{2}^{n} \mathbf{w}_{n-3} \cdots \mathbf{w}_{0}$ does not change if (for $\mathbf{w}_{0}, \ldots, \mathbf{w}_{n-3}$ fixed) $P_{2}^{n}$ is replaced by any other polygon with the same 2-dimensional volume (i.e., area) and contained in the same 2-flat. In particular, if the replacing polygon is a triangle, then the volume of the resultant simplex (and hence the original pyramid $P_{2}^{n} \mathbf{w}_{n-3} \cdots \mathbf{w}_{0}$ ) can be determined by the evaluation of a single determinant. This reduction in computational effort achieved by replacing $P_{2}^{n}$ with an equivolume triangle is the crux of the algorithm. The algorithm transforms $P_{2}^{n}$ into an equivolume $(p-1)$-gon which is in turn transformed into an equivolume ( $p-2$ )-gon and so on until an equivolume 3-gon (i.e., a triangle) is obtained. This is accomplished through the elimination of one vertex and changing the coordinates of another, with each transformation.

The algorithm for determining the volume, $c$, of the pyramid $P_{2}^{n} \mathbf{w}_{n-3} \cdots \mathbf{w}_{0}$ is presented in Figure 1 in a pseudocode language which uses matrix notation. After the code has been executed, the last 3 columns of the matrix $\mathbf{S}$ are the coordinates of the vertices of the triangle replacing $P_{2}^{n}$.

\section{FIGURE 1}

procedure content;

comment the parameters are called by value;

Local variables introduced in the algorithm:

$\mathbf{v}_{0}, \ldots, \mathbf{v}_{n-3}, \mathbf{t}_{1}, \ldots, \mathbf{t}_{p}, \mathbf{b}$ : vectors of length $n$,

S: $n \times n$ matrix,

$\mathbf{s}_{i}: i$ th column of the matrix $\mathbf{S}$,

A: $n \times 2$ matrix.

comment Step 0 - shift $\mathrm{w}_{0}$ to origin;

begin integer $i, j$;

for $i \leftarrow 0$ to $n-3$ do

begin

end;

$$
\mathbf{v}_{\mathrm{i}} \leftarrow \mathbf{w}_{i}-\mathbf{w}_{0} ;
$$

for $j \leftarrow 1$ to $p$ do

begin

end;

$$
\mathbf{t}_{j} \leftarrow \mathbf{d}_{j}-\mathbf{w}_{0}
$$

end; 
comment Step 1-initialize S;

begin

$$
\text { if } \begin{aligned}
n>3 \text { then } \mathbf{S} & \leftarrow\left(\mathbf{v}_{1}, \ldots, \mathbf{v}_{n-3}, \mathbf{t}_{1}, \mathbf{t}_{2}, \mathbf{t}_{3}\right) \\
\text { else } \mathbf{S} & \leftarrow\left(\mathbf{t}_{1}, \mathbf{t}_{2}, \mathbf{t}_{3}\right) ;
\end{aligned}
$$

end;

comment Step 2-bring in $\mathbf{t}_{\mathbf{4}}$;

begin

if $p>3$ then

begin integer $i$;

$\mathbf{s}_{n-1} \leftarrow \mathbf{t}_{2}-\mathbf{t}_{4} ;$

comment Step 3 -bring in $\mathbf{t}_{5}, \mathbf{t}_{6}, \ldots, \mathbf{t}_{p}$;

if $p>4$ then

begin

for $i \leftarrow 4$ to $p-1$ do

begin

$$
\begin{aligned}
& \mathbf{A} \leftarrow\left(\left(\mathbf{t}_{1}-\mathbf{t}_{i+1}\right),\left(\mathbf{t}_{i}-\mathbf{t}_{i+1}\right)\right) ; \\
& \mathbf{b} \leftarrow \mathbf{s}_{n-1} ;
\end{aligned}
$$

Solve $\mathbf{A}\left(h_{1}, h_{2}\right)^{\prime}=\mathbf{b}$ for $\left(h_{1}, h_{2}\right)^{\prime}$;

if $h_{2} \neq 0$ then $\mathbf{s}_{n} \leftarrow \mathbf{s}_{n}+\left(1 / h_{2}\right) \mathbf{t}_{i+1}$

else $\mathbf{s}_{n} \leftarrow \mathbf{s}_{n}+\left(1 / h_{1}\right) \mathbf{t}_{i}$;

end;

end;

end;

comment Step 4;

end;

$c \leftarrow \operatorname{ABS}(\operatorname{Det}(\mathbf{S})) / n !$

3. Outline of Proof of Algorithm. This section gives an outline of the proof of the validity of the algorithm, for $n>3$. The outline is provided, for sake of brevity, instead of a fully detailed proof.

The algorithm is valid if it can be shown that

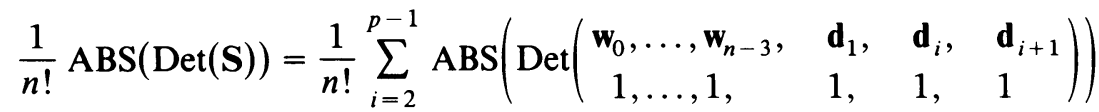

$$
\begin{aligned}
& \text { for } p \geqslant 3 \text {. }
\end{aligned}
$$

Note that the RHS of Eq. (2) is the collection of the volumes of the constituent simplices of the pyramid and $\operatorname{Det}(\mathbf{S})$ on the LHS is the value used in Step 4 of the algorithm.

Using an algebraic/geometric argument it can be shown that

$$
\begin{aligned}
\operatorname{SGN} & {\left[\operatorname{Det}\left(\mathbf{v}_{1}, \ldots, \mathbf{v}_{n-3}, \mathbf{t}_{1}, \mathbf{t}_{2}, \mathbf{t}_{3}\right)\right] } \\
= & \operatorname{SGN}\left[\operatorname{Det}\left(\mathbf{v}_{1}, \ldots, \mathbf{v}_{n-3}, \mathbf{t}_{1}, \mathbf{t}_{i}, \mathbf{t}_{i+1}\right)\right] \\
& \text { where } 3 \leqslant i \leqslant p-1, p \geqslant 4 \text { and } n>3 .
\end{aligned}
$$

It follows from the application of Eq. (3) to Eq. (2) and some algebra that it is sufficient to demonstrate that

$$
\operatorname{Det}(\mathbf{S})=\sum_{i=2}^{p-1} \operatorname{Det}\left(\mathbf{v}_{1}, \ldots, \mathbf{v}_{n-3}, \mathbf{t}_{1}, \mathbf{t}_{i}, \mathbf{t}_{i+1}\right) \text { for } p \geqslant 3 .
$$


It is a well-known fact that if $\mathbf{X}$ and $\mathbf{Y}$ are both square matrices of the same order and if $\mathbf{X}$ and $\mathbf{Y}$ differ only in their $k$ th columns (or rows), then $\operatorname{Det}(\mathbf{X})+\operatorname{Det}(\mathbf{Y})=$ $\operatorname{Det}(\mathbf{Z})$, where $\mathbf{Z}$ is a matrix whose columns (or rows) are the same as those of $\mathbf{X}$ (or Y) except that the $k$ th column (or row) is the sum of the $k$ th columns (or rows) of $\mathbf{X}$ and $\mathbf{Y}$.

It can be shown, using the above well-known fact, that the algorithm holds (i.e., Eq. (4) is satisfied) for $p=3,4, \& 5$ and then, by induction, for $p>5$.

The above is an outline of the proof of the algorithm for $p \geqslant 3$ and $n>3$. In an identical manner, it can be shown that the algorithm holds for $p \geqslant 3$ and $n=3$.

4. Efficiency of Algorithm. The steps of the algorithm, outlined in Section 2, permit us to compare its efficiency relative to other methods of volume determination. We define an "operation" as the sum of any two basic arithmetic operations $(+,-, \times, \div)$. It is assumed that all determinants are computed using Gaussian elimination with partial pivoting and require

$$
\left(\frac{n^{3}}{3}-\frac{n^{2}}{2}+\frac{2 n}{3}-\frac{1}{2}\right)
$$

operations for matrices of order $n$. Each of the methods considered utilizes the translation of $\mathbb{R}^{n}$ given in Step 0 and, as such, it will not be counted in the determination of number of operations. For the same reason, determination of $n$ ! and the division by $n$ ! will not be considered.

The volume of the pyramid of species $n-2$ can be directly evaluated by triangulation and the evaluation of the sum of the absolute values of the determinants on the RHS of Eq. (4), which excludes division by $n$ !. This requires

$$
\left((p-2)\left(\frac{n^{3}}{3}-\frac{n^{2}}{2}+\frac{2 n}{3}\right)-\frac{1}{2}\right) \text { operations. }
$$

The algorithm (Steps $1-4)$ requires

$$
\left(\left(\frac{n^{3}}{3}-\frac{n^{2}}{2}+\frac{2 n}{3}-\frac{1}{2}\right)+(p-3)\left(\frac{n}{2}\right)\right) \text { operations, for } p=3 \text { or } 4 \text {. }
$$

To perform Step 3 requires $(3 n+2)$ operations. It follows that the algorithm (Steps $1-4)$ requires

$$
\left(\left(\frac{n^{3}}{3}-\frac{n^{2}}{2}+\frac{2 n}{3}-\frac{1}{2}\right)+\frac{n}{2}+(p-4)(3 n+2)\right) \text { operations, for } p \geqslant 4
$$

Next we will consider the "repeated application of Steps 1, 2 \& 4 approach". This approach entails using Steps $1,2 \& 4$ to obtain the volumes of disjoint pairs of adjacent simplices and then summing the volumes. This method requires

$$
\left(\left[\frac{p-1}{2}\right]\left(\frac{n^{3}}{3}-\frac{n^{2}}{2}+\frac{2 n}{3}-\frac{1}{2}\right)+\left[\frac{p-2}{2}\right]\left(\frac{n}{2}\right)+\frac{1}{2}\left[\frac{p-3}{2}\right]\right) \text { operations }
$$

where, in this instance, $[\cdot]$ denotes "greatest integer in" (e.g., $[2]=2,[-7 / 3]=-3)$. Table I compares the efficiency of the three aforementioned methods of volume determination for pyramids of species $n-2$. It shows that direct evaluation is as efficient as the other two methods only when the pyramid of species $n-2$ is a simplex. Examination of Table I as well as Eqs. (5)-(8) reveals that as both $n$ and $p$ 
grow, the algorithm (Steps 1-4) becomes increasingly more efficient than the other two methods.

\section{TABLE I}

Most efficient method for determining volume of a pyramid of species $n-2$ for given $n$ and $p$

\begin{tabular}{|c|c|c|c|}
\hline \multicolumn{1}{|c|}{$p$} & $n=3$ & $n=4$ & $n \geqslant 5$ \\
\hline \hline 3 & $*$ & $*$ & $*$ \\
\hline 4 & $* *$ & $* *$ & $* *$ \\
\hline 5 & III & II & II \\
\hline$\geqslant 6$ & III & III & II \\
\hline
\end{tabular}

\begin{tabular}{|c|c|}
\hline Notation & Method of volume determination \\
\hline I & Direct Evaluation \\
\hline II & Algorithm (Steps 1-4) \\
\hline III & $\begin{array}{l}\text { Repeated Application of } \\
\text { Steps } 1,2 \& 4\end{array}$ \\
\hline * & I, II and III are equivalent \\
\hline$* *$ & $\begin{array}{l}\text { II and III are both most } \\
\text { efficient }\end{array}$ \\
\hline
\end{tabular}

Equations giving number of operations

5. Application of Algorithm for Determination of Volume of Convex Polyhedra. As noted earlier, the Cohen-Hickey method [1] for determining the volume of a convex polyhedron is by triangulation and summing the volumes of the constituent simplices. While this is correct, closer examination reveals that the convex polyhedron is partitioned into disjoint pyramids of species $n-2$ each with a 2-dimensional bounded convex polygon for a base ( $e_{i}^{2}$ in the notation of [1]); then the bases are triangulated which leads to a triangulation of each pyramid of species $n-2$ which, in turn, leads to a triangulation of the entire convex polyhedron.

To improve the efficiency of the Cohen-Hickey method, the following modification is suggested:

(i) Determine the volume, by the method indicated in Table I, of each constituent pyramid of species $n-2$. The successive adjacent vertex information can be obtained from the edges $\left(e_{j}^{1}\right)$ of a given $e_{i}^{2}$.

(ii) Sum the volumes of the pyramids of species $n-2$ to obtain the volume of the convex polyhedron.

Note that the Cohen-Hickey method, without the above modification, is equivalent to the direct evaluation method for a pyramid of species $n-2$, from a methodology and efficiency point of view.

Von Hohenbalken's method [3], [4] can be modified in an identical manner to that suggested for the Cohen-Hickey method to obtain the $n$-dimensional volume of a convex polyhedron. The algorithm (of Section 2) can easily be adapted to obtain the 
$f$-dimensional volume (where $2 \leqslant f<n$ ) of a pyramid of species $f-2$, in $\mathbb{R}^{n}$, and then applied to $f$-dimensional convex polyhedra to compute their $f$-dimensional volume.

It is perceived that considerable computational effort can be saved by selecting $\mathbf{w}_{0}$ such that it lies in more $(n-1)$-dimensional faces of the convex polyhedron than any of the other vertices. $w_{0}$ is the same for each of the constituent pyramids of species $n-2$.

6. Summary and Open Questions. An algorithm for determining the volume of a pyramid of species $n-2$ was given. It was demonstrated that the algorithm becomes increasingly more efficient than other methods, as both $n$ and $p$ increase. The algorithm was applied to the Cohen-Hickey method to improve the latter's efficiency.

The algorithm "transforms" a given pyramid of species $n-2$ into a simplex to facilitate the volume determination. Can a general $n$-dimensional convex polyhedron be efficiently "transformed" into a simplex of equal volume? Can a general $f$-dimensional convex polyhedron (where $f<n$ ) be efficiently "transformed" into an $f$-dimensional simplex* of equal volume?

Resolution of the above questions may lead to more efficient procedures for volume determination. The author is exploring these possibilities.

Acknowledgments. The author is grateful to Professor Victor Klee, of the Department of Mathematics at University of Washington (Seattle, Washington), for pointing out a number of references in the literature and Professor J. Michael McNamee, of the Department of Computer Science at York University (Toronto, Canada), for constructive criticism and assistance relative to presentation of the material in the final manuscript. The author is also indebted to the referees for many helpful comments and suggestions.

Corporate Productivity Department

Bell Canada

Bell Trinity Square

Toronto, Ontario, Canada M5G 2E1

1. J. Cohen \& T. Hickey, "Two algorithms for determining volumes of convex polyhedra," J. Assoc. Comput. Mach., v. 26, 1979, pp. 401-414.

2. D. M. Y. Sommerville, An Introduction to the Geometry of N Dimensions, Dover, New York, 1958.

3. B. Von Hohenbalken, Research Paper No. 79-17, How to Simplicially Partition a Polytope. Presented at the Tenth International Symposium on Mathematical Programming, Montreal, Canada, August 1979.

4. B. Von Hohenbalken, "Finding simplicial subdivisions of polytopes," Math. Programming, v. 21, 1981, pp. 233-234.

*An $f$-dimensional simplex has $f+1$ vertices, in $\mathbf{R}^{n}$, which define a unique $f$-flat. 